\title{
Online Monitoring System for Track Slab Temperature
}

\author{
Wenfa Zhu ${ }^{1 \mathrm{a}}$, Huizhen $\mathrm{Ma}^{2 \mathrm{~b}}$, Haomin $\mathrm{Liu}^{1}$,Wenbing Yang ${ }^{1}$ \\ ${ }^{1}$ School of Urban Railway Transportation, Shanghai University of Engineering and Technology, \\ Shanghai, China; \\ ${ }^{2}$ Library, Shanghai University of Engineering and Technology, Shanghai, China \\ a'Wenfa Zhu zhuwenfa1986@163.com, bHuizhen Ma 714434654@qq.com
}

Keywords: Track Slab; Monitoring; Temperature; Operation Security

\begin{abstract}
Under the action of temperature, the change of temperature gradient exists in the vertical direction of the track slab in its structure. The temperature gradient will arouse the bending deformation between the track slab and the mortar cushion, which will result in the separation of the track slab layer and even have a certain influence on the safe operation of the train. In the paper, a set of online monitoring system for track slab temperature is developed, which realizes online acquisition track slab temperature data and real-time warning. The system is composed of three parts: the front-end acquisition hardware, the back-end processing and analysis center, and the user terminal. Because of stable high-precision acquisition, remote transmission and reporting of detection results, and longtime solar power supply the system has been successfully used for monitoring track slab temperature of railway passenger line in east China. Real-time monitoring the track slab temperature change is very important to improve the design of rail structure, disease prevention and maintenance.
\end{abstract}

\section{Introduction}

The track slab is composed of thermal conductivity and poor heat transfer performance concrete material, under the action of temperature load it could lead directly to the serious nonlinear temperature distribution [1-3], which results in warping deformation and affects the smoothness of the rail and endangers the driving safety. Therefore, the research of track slab temperature is the focus in the field of evaluation of orbital structural energy. Under different climatic conditions, Liu $\mathrm{Yu}$ [4] observed the track slab temperature in the field of the CA mortar grouting, measured the temperature gradient of the track slab and calculated the temperature warping deformation and stress of the track slab under the measured temperature gradient. Yang Jingjing et al. [5] measured the temperature of the track slab under open-air environment and simulated the temperature field of the track slab by loading the temperature loading of the joint. The temperature deformation of the track slab under the combined action of the whole temperature rise and the temperature gradient of the track slab is analyzed. However, it is no report that real-time acquisition and uploading track slab temperature data can be accomplished at the same time in the high-speed rail environment with no power supply, high pollution, high electromagnetic interference, large temperature difference between day and night. Therefore, the paper develops a system to monitor the track slab temperature in high-speed rail environment. Base on this system, we can study the change of structure temperature of the track slab under ambient temperature, understand the track slab service state and provide scientific suggestions for the design, disease prevention and maintenance of the rail structure.

\section{System Overall Design}

As is shown in Figure 1, the system is mainly composed of portable data acquisition hardware, processing and analysis center and user terminal. NI CompactDAQ-9188XT, NI data acquisition card NI9205 and so on are used for building the data acquisition hardware to obtain the track slab 
temperature. By 4G mobile network the temperature data is sent to the processing and analysis center , in the processing and analysis center data analysis and early warning is accomplished by the system software written in LabView[6].Various test parameters Settings and start-stop instructions is sent out by wireless terminal equipment such as laptop, tablet, Smartphone, etc. The mass data is automatically processed and analyzed by the system, and the results back to the user terminal..
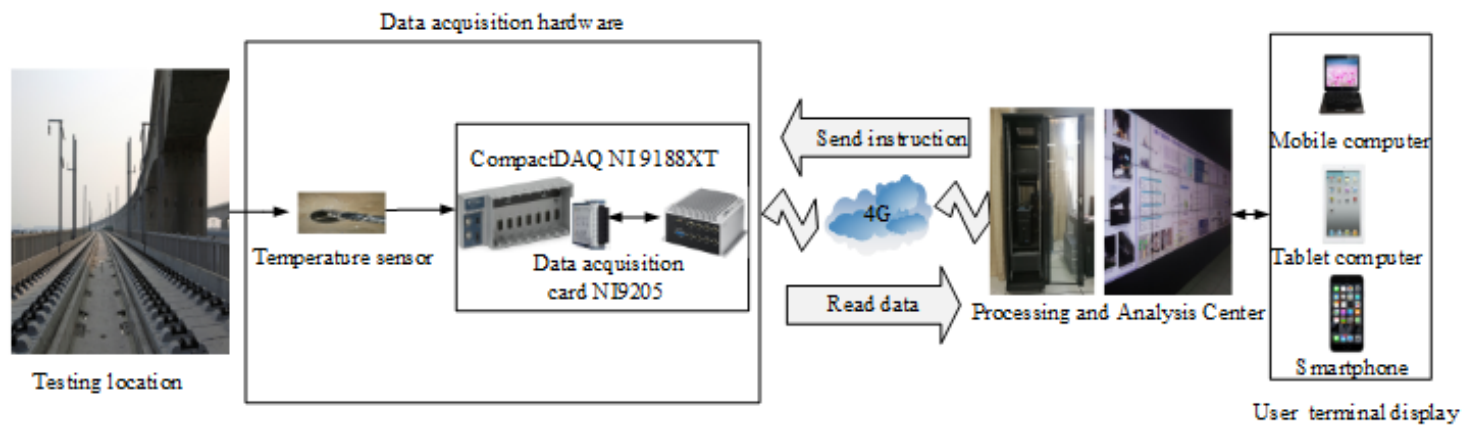

Fig. 1 System schematic diagram

\section{Front-end data acquisition hardware design}

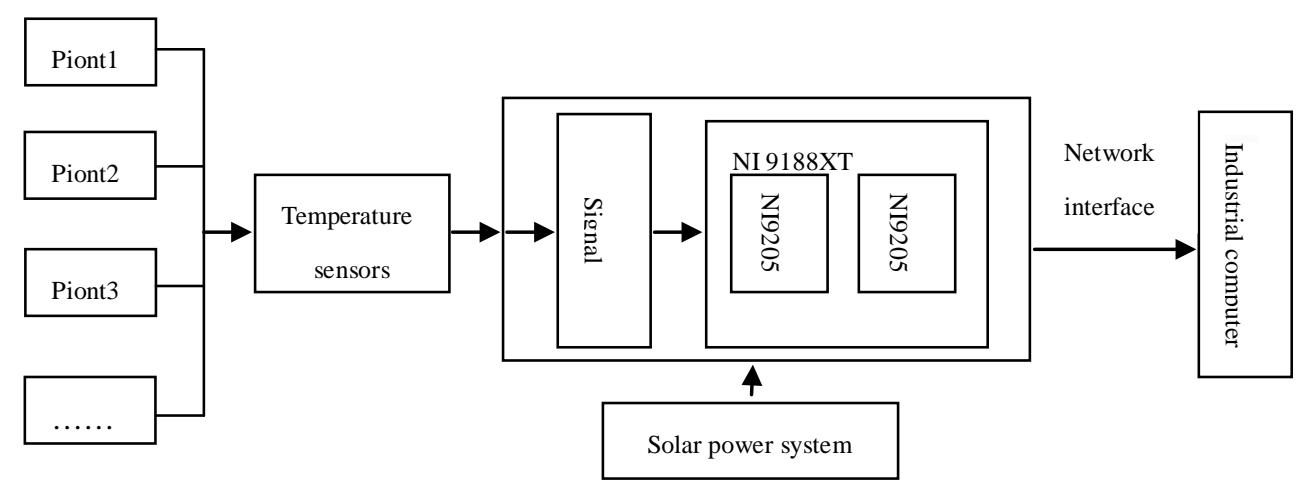

Fig. 2 The front-end acquisition hardware structure diagram

As is shown in Figure 2, the front-end data acquisition hardware is composed of the NI CompactDAQ-9188XT, the data acquisition card NI9205 and the temperature sensor to achieve stable high-precision data acquisition of the track slab temperature.

As is shown in Fig. 3, the data acquisition card NI9205 collects the temperature data signal from the sensor which is embedded into the track slab, then the high-speed data communication is realized through the network interface of NI CompactDAQ-9188XT.

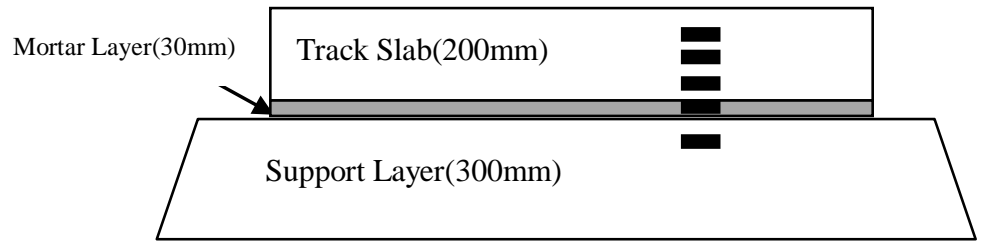

Fig. 3 Measurement Points layout

Stable and reliable power supply system is the key of long-term online monitoring of track slab temperature. However, conventional solar energy supply mode often lacks effective management strategy, which not only leads to waste of energy but also increases system maintenance cost. In this paper, the solar power supply and management system shown in Fig. 4 is designed for the online monitoring of the track slab temperature, and the solar energy is recycled and stored in the lithium battery pack through the photovoltaic machine. The fuzzy generalized maximum power Point tracking control strategy is adopted to realize the charge and discharge management of the energy storage unit at the same time, which can effectively improve the efficiency of solar power and prolong the service life of the lithium battery. 


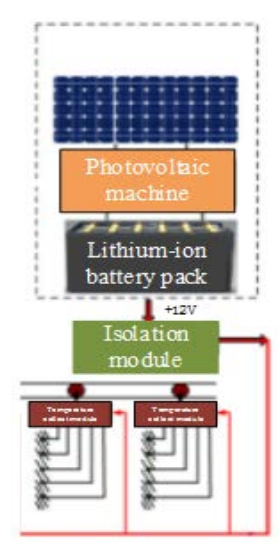

Fig. 4 Solar power supply system structure diagram

\section{Software Design}

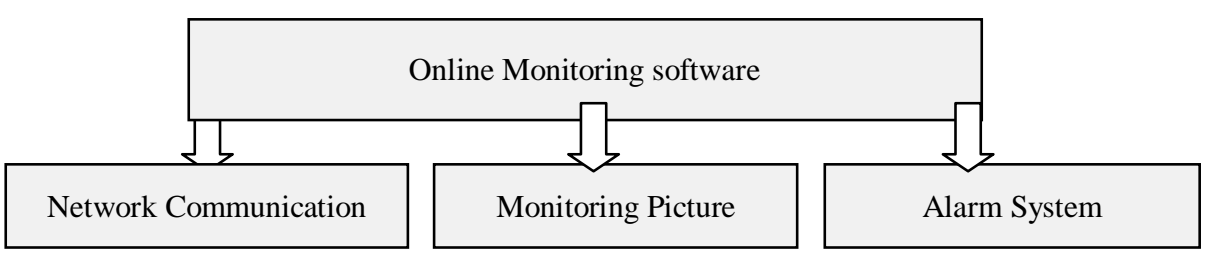

Fig. 5 Software system composition diagram

Track slab temperature online monitoring software is mainly used for data acquisition, transmission, processing, storing and display functions. As is shown in Figure 5, according to the monitoring system functional requirements, online acquisition software mainly includes network communication, monitoring picture and alarm system. The online monitoring module is questions and answers mode, which means that the upper machine sends the hardware instructions to the lower computer, which immediately returns the data to the upper machine after receiving instruction. The upper computer provides the user for intuitive monitoring data by judging and analysis the data [6]. The data monitoring sequence diagram is shown in Figure 6.

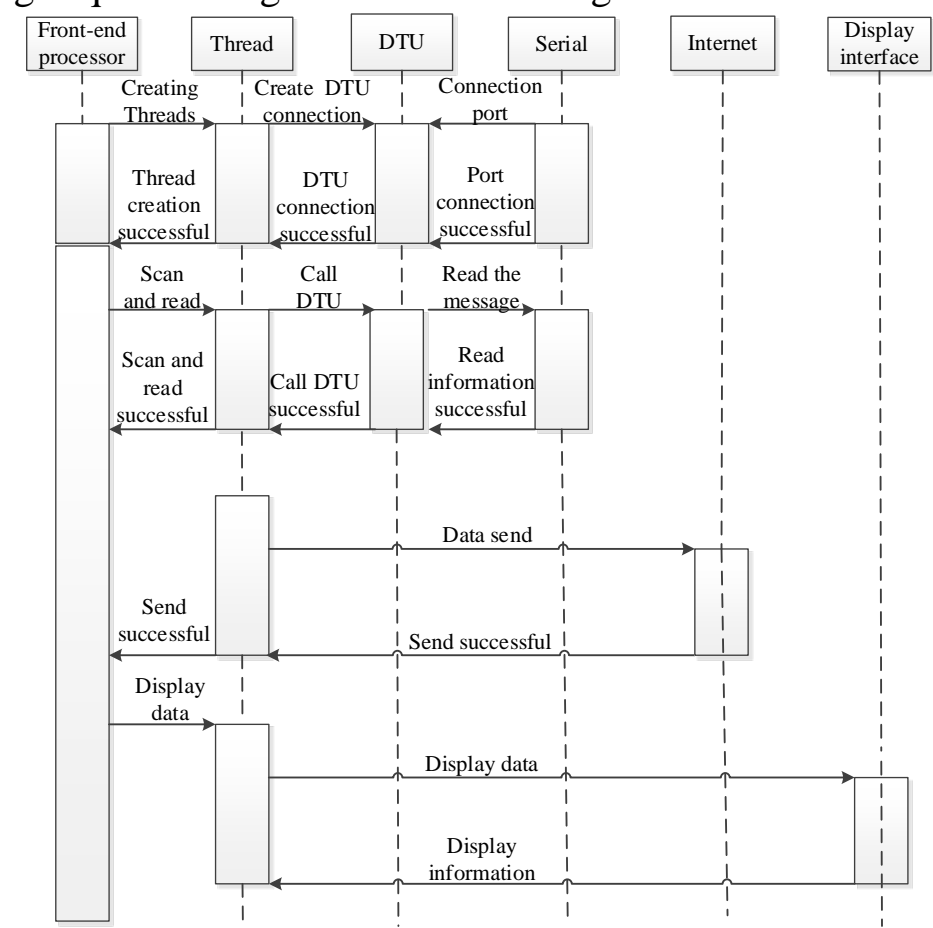

Fig. 6 Data monitoring sequence diagram 


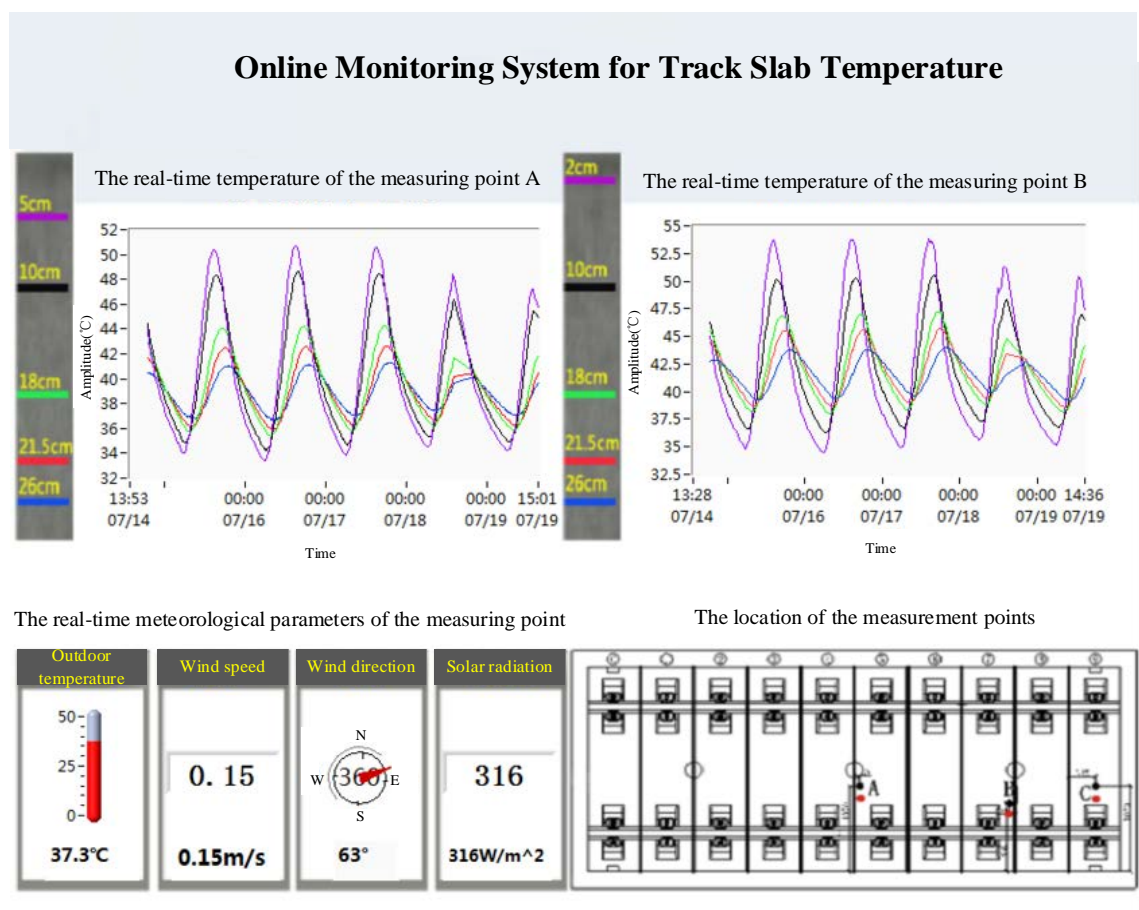

Fig. 7 Monitoring interface

This paper realizes the security early warning and the remote online management of the rail board temperature data, which provides data support for the maintenance key time node of the track slab by the combination of data analysis and theoretical analysis. As is shown in Fig. 7, the monitoring interface in the processing and analysis center is real-time temperature and meteorological parameters of the track slab of a railway passenger line in east China in the summer .

\section{Conclusion}

The temperature is collected from track slab by the on-line monitoring system, in the processing and analysis center the monitoring screen display the real-time track slab temperature which is transmitted by 4G mobile network from the measurement points, after data analysis the exceed-limit alarm can be given. The data analysis results can provide directional guidance and scientific advice for design improvement, disease prevention and maintenance of rail structure. With the 'Internet +' era and the advent of 'Industrial 4.0', the development of intelligent testing system has become an irresistible trend, the system will provide real-time data for the rail department and provide a high-efficiency maintenance and monitoring means for the rail workers. At present, the system has been successfully used in online monitoring for the track slab temperature of a passenger line in east China, along with the rapid development of rail transit in our country, this system has a very broad prospect of engineering application.

\section{Acknowledgement}

In this paper, the research was sponsored by the Nature Science Foundation (Project No.51478258), Key Technology R\&D Project of Shanghai Committee of Science and Technology (Project No. 16030501400), the research and development of science and technology of Shanghai Railway Bureau (Project No. 20160285)

\section{References}

[1] Gao Liang, You Mingxi, Xiao Hong et al. Experimental study on the relationship between 
temperature distribution and ambient temperature of SRTS II slab ballastless track [J]. Railway Construction .2016 (05): 15-18.

[2] Zhang Chong Wang, Su Chengguang, Liu Xueyi, et al.Effects of Temperature Gradient and Subplate Void on Dynamic Response of Train Track System [J]. Journal of the China Railway Society, 2010 (04): 482-486.

[3] Xu Qingyuan, Fan Hao, Li Bin. Influence of temperature gradient load of ballastless track on dynamic characteristics of slab ballastless track system on trains - subgrade [J]. Journal of Railway Science and Engineering, 2013,10 (3): 1-6.

[4] Liu Yu, Chen Pan, Zhao Guotang. Study on early temperature field characteristics of CRTS II plate - type ballastless track structure [J]. China Railway Science, 2014,35 (1): 1-6.

[5] Yang Jingjing, Zhang Nan, Gao Mengmang and so on. (3): 210-217 (in Chinese) [J]. Engineering Mechanics, 2016,33 (4): 210-217.

[6] Zhu Wenfa, Chai Xiaodong, Zheng Shubin, et al.Design of signal acquisition and processing system for inertial measurement unit based on LabVIEW [J]. Computer Measurement and Control, 2012,20 (6): 1697-1698. 